\title{
Finding the range to a distant object near the sea surface
}

\author{
Marianne A. C. Degache ${ }^{\mathrm{a}}$, Stephen Hammel ${ }^{\mathrm{b}}$ \\ ${ }^{a}$ TNO Defence, Security and Safety, The Hague, The Netherlands \\ ${ }^{\mathrm{b}}$ Space and Naval Warfare Systems Center, San Diego CA
}

\begin{abstract}
Within the marine atmospheric surface layer it is possible for a single camera to deduce passively the range to a point target. Although this range determination would appear impossible at first glance, such a measurement exploits the common occurrence of sub-refractive propagation conditions in the marine environment.

A calculation of the range to an object utilizes a geometric optics determination of slight angular differences between two different ray trajectories to the object. This is most commonly done with the assumption of Euclidean or 'free-space' conditions. In this paper we utilize the phenomenon of inferior mirages to provide two different ray-paths to an imaging sensor. The primary assumption is that the environment containing the path from camera (or eye) to target is homogeneous (but not isotropic).
\end{abstract}

Keywords: passive ranging, marine environment, geometrical optics

\section{INTRODUCTION}

Modern surface Navy ships require dependable and predictable communications, surveillance, and tracking systems. These systems in turn require a capability to predict the electromagnetic propagation environment. In this paper we are concerned with models for infrared and visible propagation.

Although the full atmospheric hemisphere surrounding the ship must be characterized, the most critical portion is the 100 meter thick surface layer containing the ship and extending to the horizon. This determination of the propagation environment for surface ships can be a difficult problem. Extended horizontal propagation paths in the atmospheric surface layer encounter relatively dynamic refractivity conditions. These conditions have been investigated with a combination of model development and field experiments. This paper will describe the application of a geometrical optics model to analyze the near-surface maritime atmospheric propagation environment.

\section{MODELS FOR REFRACTIVE PROPAGATION}

There are two different models that we will use for characterizing the propagation environment: IRWarp ${ }^{1}$, and EOSTAR $^{2}$. The primary computational tool for analysis of refractive effects in both of these models is a geometrical optics tool that computes a ray-trace calculation for a given refractive environment. The ray-trace data is utilized to generate detailed information about geometrical transformations induced by the propagation environment.

The geometrical optics ray-trace method is initiated by a definition of the local refractivity field. We assume that within the marine atmospheric surface layer this field can be considered to be horizontally homogeneous. For optical and infrared frequencies, the primary determinant of the refractivity profile is the vertical temperature profile.

\subsection{The Vertical Temperature Profile}

The generation of a continuous vertical temperature profile is based upon a surface layer similarity theory developed by Monin and Obukhov. IRWarp uses the model formulation based upon bulk methods for calculating turbulence parameters described by Frederickson et al. [1]. The profile is defined in terms of the potential temperature $\theta$ which is

\footnotetext{
${ }^{1}$ IRWarp is an IDL widget that computes a geometrical optics ray-path. It is designed to model the near-surface marine atmospheric surface layer.

${ }^{2}$ EOSTAR (Electro-Optical Signal Transmission And Ranging) is an EO sensor performance model. It predicts the performance of imaging sensors in a maritime environment.
}

Atmospheric Optics: Models, Measurements, and Target-in-the-Loop Propagation,

edited by Stephen M. Hammel, Alexander M. J. van Eijk, Michael T. Valley, Mikhail A. Vorontsov,

Proc. of SPIE Vol. 6708, 67080U, (2007) · 0277-786X/07/\$18 · doi: 10.1117/12.741056

Proc. of SPIE Vol. 6708 67080U-1 
derived from the air temperature $T$ by $\theta=T+\Gamma_{d} z$ where $\Gamma_{d}$ is the dry adiabatic lapse rate, and $z$ is height above the surface. Air temperature measurements are taken at the sea surface, and at a reference height. These sparse data are then used in a bulk model to generate a continuous vertical profile, and with a sea surface temperature given by $\theta_{0}=T_{0}$, the temperature $\theta(z)$ at a height $z$ above the water surface is given by:

$$
\theta=\theta_{0}+\frac{\theta_{*}}{k}\left[\ln \left(z / Z_{0 \theta}\right)-\Psi_{\theta}(z / L)\right]
$$

Where $Z_{0 \theta}$ is the roughness length for the temperature profile, $\theta_{*}$ is the potential temperature scaling parameter, and $\theta_{0}$ is the surface potential temperature. $L$ is the Monin-Obukhov length, and $\Psi_{\theta}(z / L)$ is a stability correction function.

\subsection{The Refractivity Gradient and Ray Curvature}

The optical path calculation for a ray propagating in the atmosphere requires a determination of the refractivity field. The near sea surface atmosphere is assumed to be horizontally homogeneous. The vertical structure is divided into layers, and for each layer a refractivity gradient can be determined. Our approach follows the work of Lehn [2].

A ray propagating through a medium with a gradient in refractive index will define a curved path. The refractive index $n$ is found:

$$
n=1+\alpha(\lambda) \frac{P}{T}
$$

where $\alpha(\lambda)=\left(77.6+0.584 \lambda^{-2}\right) \times 10^{-6}$ and $\lambda$ is the wavelength in micrometers. Hence the refractive gradient is:

$$
\frac{d n}{d z}=\frac{\alpha(\lambda)}{T^{2}}\left(T \frac{d P}{d z}-P \frac{d T}{d z}\right)
$$

The hydrostatic equation gives

$$
\frac{d P}{d z}=-g \rho=-g \beta \frac{P}{T}
$$

for density $\rho$ and gravitational acceleration $g$, and $\beta=3.485 \times 10^{-3}$. Hence

$$
\frac{d n}{d z}=-\frac{\alpha(\lambda) P}{T^{2}}\left(g \beta+\frac{d T}{d z}\right)
$$

The curvature $\kappa$ of a ray is defined such that positive curvature means a ray is concave towards the earth:

$$
\kappa=-\frac{\sin (\xi)}{n} \frac{d n}{d z}
$$

$\xi$ is the angle between the ray and the normal to the local surface tangent plane. The radius of curvature $r$ is $r=1 / \kappa$ and this yields

$$
r=-\frac{n T^{2}}{\sin (\xi) \alpha(\lambda) P(g \beta+d T / d z)}
$$


where $r>0$ defines a ray that is concave down toward the earth.

For a ray-trace calculation the atmosphere is divided into concentric shells of constant thickness and constant height. Within each shell or layer, a refractivity gradient is defined as a function of the temperature gradient. We take the temperature gradient within the layer to be constant as well, and therefore the radius of curvature for each layer is a constant.
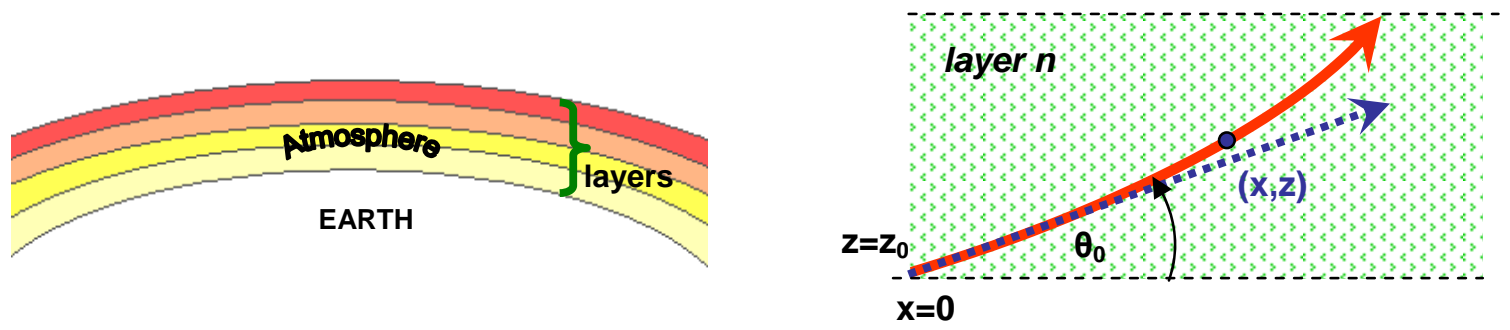

The ray-trace procedure proceeds by determining the entry height $z_{0}$ and entry angle $\phi_{0}$ of a ray into a layer, where $\phi_{0}$ is the angle with the horizontal. The coordinate system is established to put the entry point at range $x=0$ and height $z=z_{0}$. The equation for the ray trajectory in this layer is

$$
z=-\frac{x^{2}}{2 r}+x \tan \phi_{0}+z_{0}
$$

The algorithm described above requires an assumption of piecewise horizontal homogeneity of the refractive field: the only gradients in refractive index occur in the vertical coordinate. The assumption of piecewise horizontal homogeneity means that the computation of the ray-trace can be confined to a vertical plane. In the remainder of this paper we will restrict further to an assumption of horizontal homogeneity. The analysis is performed in this two-dimensional plane and the coordinate system is defined with the origin at mean sea level so that the receiver location is specified by the coordinates $\left(0 ; z_{r}\right)$ and the transmitter is at $\left(x_{t} ; z_{t}\right)$. The local coordinate system is further transformed to bring the curved surface of mean sea level to a horizontal plane (a "flat earth" representation). Thus the $\mathrm{x}$-axis in the two dimensional representation is the mean sea surface, and range information along the propagation path is measured in this coordinate. The z-axis represents the vertical offset from $z=0$ at mean sea level.

\section{SUB-REFRACTIVE MIRAGES ARE COMMON IN THE MARINE ENVIRONMENT}

Although some of the analysis presented in this paper applies to general atmospheric conditions, we will concentrate on the sub-refractive conditions. These conditions occur essentially when the air temperature at some small height above the surface is less than the sea temperature: $\left(T_{\text {air }}-T_{\text {sea }}<0\right)$. To get a rough idea of the probability of occurrence of $T_{\text {air }}-T_{\text {sea }}<0$, a survey of $8,088,855$ shipboard measurements from the Ducting Climatology Survey over 273 Marsden squares worldwide reveals that $T_{\text {air }}-T_{\text {sea }}<0$ for more than $89 \%$ of the samples. The remainder of this section develops a result for passive ranging given the existence of sub-refractive conditions.

The ray-trace algorithm first defines the vertical profile as a set of discrete layers, each with a characteristic temperature and refractivity gradient. A characteristic radius of curvature is then assigned to each layer using eqn. (6) above. The conditions for a sub-refractive mirage require a surface temperature relatively warmer than the air temperature a short distance above the surface. Monin-Obukhov similarity theory can be applied to deduce the vertical temperature profile for this situation, since the Richardson number is negative and unstable conditions apply. The form of the vertical temperature gradient given by similarity theory is

$$
\frac{d T}{d z} \propto \ln \left(\frac{z}{z_{0}}\right)
$$


where $z$ is the height above the surface. Thus $d T / d z$ has a large negative value very near the surface and it increases toward zero as height increases.

Considering a fixed value of $\lambda$ and a constant air pressure, the pressure variations as a function of height can be neglected for the geometry of interest, $(0 \leq z \leq 50$ meters), so eqn. (4) can be written

$$
\frac{d n}{d z}=\frac{-C_{0}}{T^{2}}\left(g \beta+\frac{d T}{d z}\right)
$$

This shows that $d T / d z$ is large and positive very near the surface, and that it decreases monotonically as $z$ increases.

The vertical temperature profile defines a set of layers $\sigma_{1} \ldots \sigma_{u}$ with $\sigma_{1}$ denoting the layer nearest the surface. A temperature and a height are determined for each layer boundary, and associated with each layer $\sigma_{i}$ is a vertical thickness $\Delta z_{i}$. Within each layer $\sigma_{i}$ there is an associated temperature gradient $\nabla T_{i}=(d T / d z)_{z \in \sigma_{i}}$. From this we determine a refractivity gradient $\nabla N_{i}=(d n / d z)_{z \in \sigma_{i}}$, and finally by the calculation in eqn. (6) an associated radius $r_{i}$. For sub-refractive conditions, $T_{1}>T_{2}>\ldots T_{u}$, because of eqn. (8) and eqn. (9)

$$
\begin{gathered}
\left|\nabla N_{1}\right|>\left|\nabla N_{2}\right|>\ldots\left|\nabla N_{k}\right| \\
\left|r_{1}\right|<\left|r_{2}\right|<\ldots\left|r_{u}\right|
\end{gathered}
$$

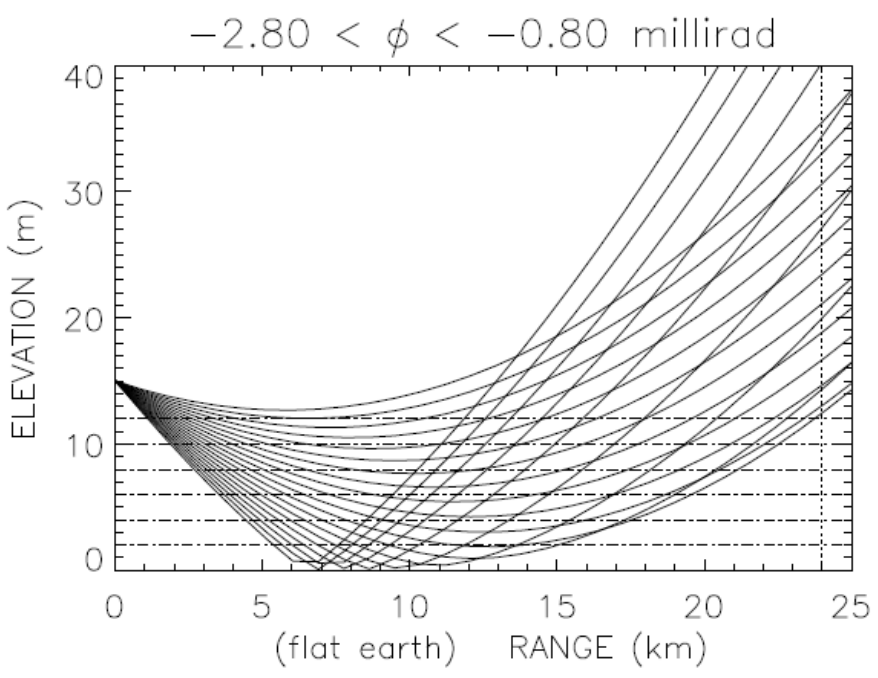

Fig. 1. The vertical scale is in meters above the surface, while the horizontal scale is in kilometers downrange from the sensor which is at a height of 15 meters. The 6 horizontal dashed lines indicate constant surfaces of 2, 4, 6, 8, 10, and 12 meters. The rays that appear to be reflecting from the $\mathrm{x}$-axis are actually refracted.

Since $d n_{i} / d z<0$ and $r_{i}<0$ for all the layers, all rays will be concave upward. The implication for a ray calculation in sub-refractive conditions is that the radius of curvature of a ray decreases as the height above the surface decreases. 
In order to examine the intersection structure of the rays, we will be primarily concerned with rays for which the entry angle $\phi_{0}<0$. These rays initially decrease in height as range increases. As fig. 1 indicates, we are interested in a fan of rays initiated at a single point $S=\left(0 ; z_{s}\right)$ with a range of entry angles. Assume we have a fan of rays $\left\{\rho_{j}\right\}_{j=1, \ldots, m}$ with each ray $\rho_{j}$ emanating from the receiver location $R=\left(0 ; z_{r}\right)$. Each ray $\rho_{j}$ has an initial entry angle $\phi_{j}(0)$ (measured from the horizontal) and the ray ordering implies that $\phi_{1}(0)<\ldots<\phi_{m}(0)<0$. We also require of the fan of rays that there is a constant angular difference between successive rays: $\delta \phi=\phi_{j}(0)-\phi_{j-1}(0)$.

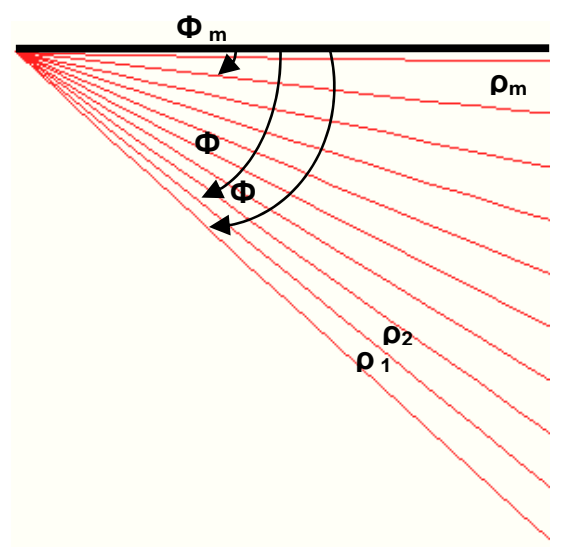

We now consider the lowest point on a ray $\rho_{j}$. Let $\hat{z}=\min \left\{z:(x, z) \in \rho_{j}\right\}$. There are two possible ray types: rays that decrease in height and have a single minimum and thence monotonically increase in height above the surface $(\hat{z}>0)$, and rays that continue to descend and terminate at the surface $(\hat{z}=0)$. We consider only those rays for which $\hat{z}>0$, and label the point of minimum height of ray $\rho_{j}$ as $v_{j}=\left(\hat{x}_{j}, \hat{z}_{j}\right)$. We require that the fan of rays be selected so that ray $\rho_{0}$ terminates at the surface $z=0$, and additionally we require that the full angle of the fan of rays is restricted such that all rays satisfy $\hat{x}_{j}>\hat{x}_{1}$.

Finally we assume that if two rays $\rho_{j}$ and $\rho_{k}$ intersect a level surface at height $z_{j}$ at $\left(x_{j}, z_{j}\right)$ and $\left(x_{k}, z_{k}\right)$ respectively, if $\phi_{j}<\phi_{k}<0$, then the minima $\left(\hat{x}_{j}, \hat{z}_{j}\right)$ and $\left(\hat{x}_{k}, \hat{z}_{k}\right)$ satisfy $\hat{z}_{j}<\hat{z}_{k}$. We make the following two assertions which will not be proved:

Assertion 1 If the two rays $\rho_{j}$ and $\rho_{k}$ satisfy the above conditions, and in addition $x_{j}=x_{k}$, then there will be an intersection $\rho_{j} \cap \rho_{k}=\left(x_{T}, z_{T}\right)$ with $x_{T}>\hat{x}_{j}$.

Assertion 2 Reciprocity: The ray is symmetric about the minimum point $\left(\hat{x}_{j}, \hat{z}_{j}\right)$.

A ray with a more negative downward launch angle will necessarily enter a region for which the radius of curvature is smaller in magnitude (and negative, hence concave upwards). The reciprocity property organizes the fan of rays. For the fan $\left\{\rho_{j}\right\}_{j=1, \ldots, m}$ and the associated set of minima $\left\{v_{j}\right\}_{j=1, \ldots, m}$ with $v_{j}=\left(\hat{x}_{j}, \hat{z}_{j}\right)$, we have $\hat{x}_{1}<\hat{x}_{2}<\ldots<\hat{x}_{R}$ with $0<R \leq m$. For $j=1, \ldots, R$ and $k=1, \ldots, m$ we have $\rho_{j} \cap \rho_{k} \neq \varnothing$. 


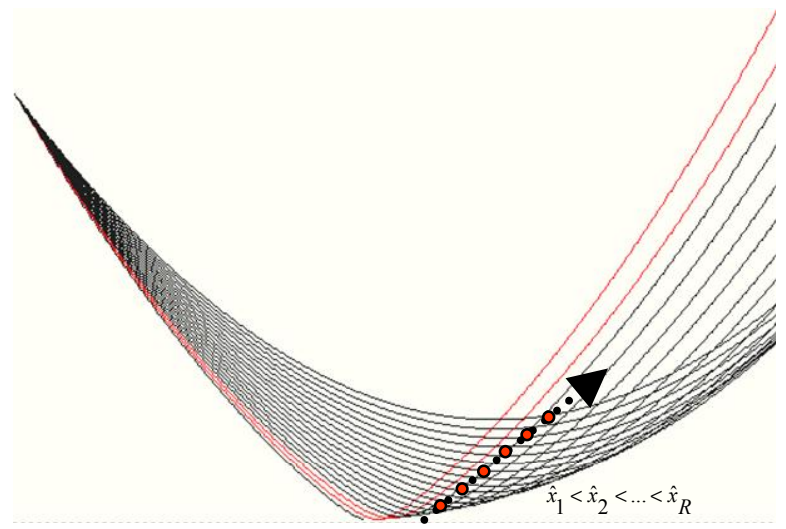

This forces the existence of an intersection between rays, and the general conditions which imply the existence of a boundary envelope of ray intersections called a caustic. Furthermore, the intersection structure is ordered, so that the set of intersections $\left(x_{1 k}, z_{1 k}\right)=\rho_{1} \cap \rho_{k}$ satisfies $x_{11}<x_{12}<\ldots<x_{1 m}$. In the next section we will show how a coordinate transformation is induced by the intersection structure of the rays.

\section{USING MULTIPLE MIRAGE IMAGES TO FIND HEIGHT AND RANGE}

During sub-refractive mirage conditions, an imaging sensor will record two distinct images of a single point source. In previous studies it has been shown $[6,7]$ that the two images can be exploited to provide both height and range information. We will now show how the ray-trace procedure within IRWarp creates a coordinate transformation.

\subsection{Using Isomets}

In fig. 1 a receiver has been positioned at a height of 15 meters, and the set of rays tracing the propagation path defines an envelope. The ray envelope has an intersection structure with a set of constant-height surfaces at heights of 2, 4, 6, 8, 10, and 12 meters. A ray traced from the receiver intersects a given constant-height surface either once, twice, or not at all. The intersection structure of the constant-height surfaces with the ray-trace envelope induces a transformation.

The result of this transformation is shown in fig. 2. We define an isomet surface as a surface of constant height, and we will use the term isomet to refer to the contour curves representing the intersection set between a constant-height surface and the ray-trace envelope shown in fig. 2. Each of the isomets in fig. 2 displays a similar form. The vertical axis shows angular displacement from the horizontal tangent plane at the sensor. The horizontal axis shows range.

The graph of a single isomet can be interpreted by imagining a source confined to one of the isomet surfaces shown in fig. 1 Consider a source on the 12 meter isomet as it moves toward the sensor from the $25 \mathrm{~km}$ range. At $\approx 23.5 \mathrm{~km}$, the source appears over the horizon as a single point which immediately splits into two images. As seen through an imaging sensor, for example, one image decreases in angular elevation, and the upper image increases in angular elevation as the source moves closer in range. This information is depicted in fig. 2 and corresponds to the outermost curve labelled " 12 $\mathrm{m}$ ". At $\approx 11.5 \mathrm{~km}$, the lower image descends below 2.8 milliradians: in terms of the imaginary sensor, it has descended beneath the lowest edge of the sensor focal plane. The (now solitary) upper image continues to rise to the upper edge of the sensor field-of-view. 


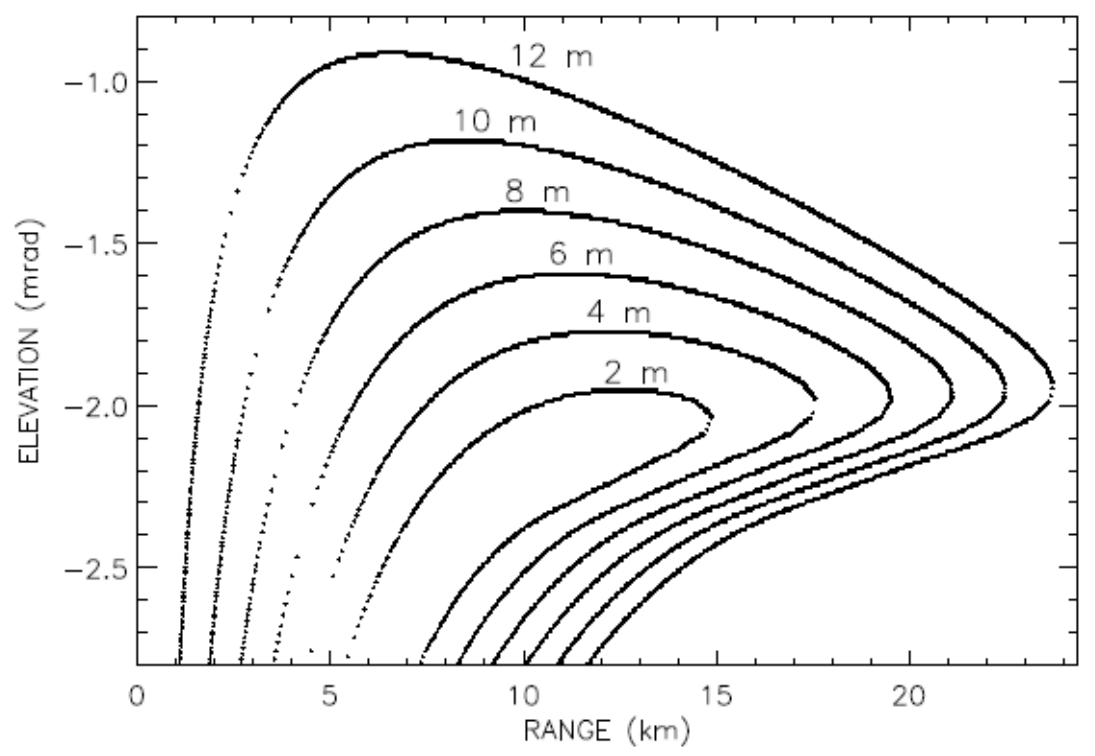

Fig. 2. A series of isomets at the heights of 2, 4, 6, 8, 10, and 12 meters. For a given range value, each isomet defines either 0,1 , or 2 corresponding elevation values.

Within the last $6 \mathrm{~km}$, the source is seen to rapidly move from near the top edge to disappear below the bottom edge. The shape of the $12 \mathrm{~m}$ isomet is characteristic of all the isomet contours for surfaces of height less than the sensor height. When the isomet surface height is greater than sensor height, an inbound upper image disappears across the upper boundary, and never re-crosses from top to bottom.

The key to a deduction of height and range from angular elevation information is the utilization of those portions of an isomet for which two values of elevation correspond to a single range value. Thus for the 12-meter isomet, ranges between $11.5 \mathrm{~km}$ and $23.5 \mathrm{~km}$ correspond to two distinct elevation values. This indicates that it is possible to find a oneto-one correspondence between a pair of elevation angles, and a height-range pair.

The actual transformation from the (lower angle, upper angle) coordinate space to the (height, range) coordinate space is shown in fig. 3. When two images are detected by a sensor, the elevations of the lower and upper images can be plotted as a point in fig. 3, and the height and range of that point can be read from the inner coordinate system. To say it differently, the figure contains the transformation which takes two elevation measurements as input, and generates as output both height and range of the source or target. In terms of coordinate systems, the rectilinear lower elevation vs upper elevation coordinate system is transformed to the distorted, curvilinear height vs range coordinate system.

Consider as an example an imaging sensor system with a telescope which detects a source in a sub-refractive mirage regime. The two elevations can be determined from the imaging frame: suppose $\left(\phi_{\text {lower }}, \phi_{\text {upper }}\right)=(-2.65,-1.6)$. The transformation displayed in fig. 3 shows the actual range and height can be read out from the transformed coordinate system yielding range $\approx 10 \mathrm{~km}$, and height $\approx 6 \mathrm{~m}$. 


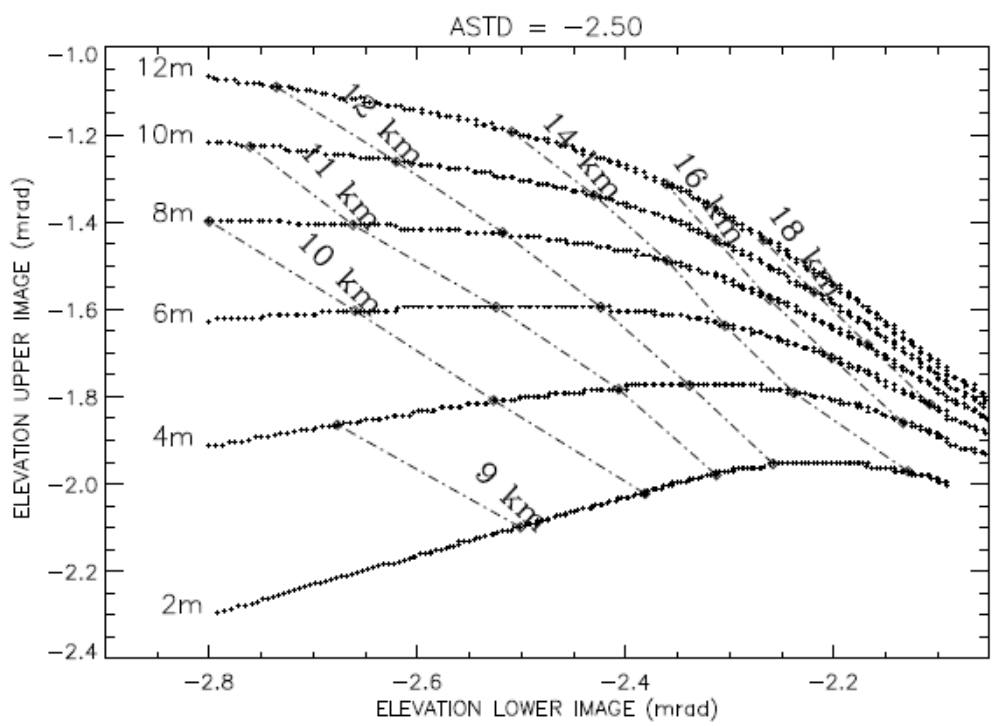

Fig. 3. The transformation that is implied by the data in fig. 2. The same information is shown here, but restricted to the portions of the isomets that are dual-valued. For example, the angular elevation pair $\left(\phi_{\text {lower }}, \phi_{\text {upper }}\right)=(-2.65,-1.6)$ transforms to a range $\approx 10 \mathrm{~km}$ and a height $\approx 6 \mathrm{~m}$

\subsection{Potential for Application}

As we noted above, sub-refractive conditions are quite common for the marine atmospheric surface layer. For our imaging geometry, these conditions cause mirages that appear at two different elevations. The usable range for the particular example presented here is from $9 \mathrm{~km}$ out to $\approx 18 \mathrm{~km}$. Note that the range limits for effective range-finding are determined by the intensity of the sub-refractive conditions and by sea-surface roughness. As air-sea temperature difference $T_{\text {air }}-T_{\text {sea }}$ becomes more negative, the range domain for which two images occur increases in extent by moving the point of first appearance of two images closer to the sensor. Conversely, as air-sea temperature difference $T_{\text {air }}-T_{\text {sea }}$ becomes less negative and closer to zero, the range domain for which two images occur decreases in extent: the first appearance of two images occurs at a point further away from the sensor. A rough sea surface will occlude the lowest height rays which will also force the point of first appearance of mirages further away from the sensor.

\section{THE TRANSFER MAP}

An important application of the geometric optics approach is the calculation of the observable effects of refractive gradients. A fundamental problem is to predict the image of a source or target at a given range. This information is accessible through the calculation of a transfer map within the EOSTAR and IRWarp models and we will describe this calculation along with some of its applications.

A bundle of rays is defined at a common point at the receiver to span the vertical extent of the sensor field-of-view (fig. 1). Each ray $\rho_{\text {refract }}$ is generated within the vertical plane containing the transmitter and receiver starting from the receiver location at $\left(0 ; \mathrm{z}_{\mathrm{r}}\right)$. Launch angles $\phi_{\text {refract }}$ are defined with respect to the ray based at $\left(0 ; \mathrm{x}_{\mathrm{r}}\right)$ parallel to the $\mathrm{x}$ axis. If the source point is visible (at infrared frequency) to the receiver, we can be certain that a fan of rays defined for launch angles $-\pi / 2 \leq \phi_{\text {refract }}<\pi / 2$ will include rays that intercept the source. In practice, the computational angular extent of the fan of rays is further constrained since rays launched with a sharp downward angle will intercept the $\mathrm{x}$-axis (earth surface) before the source range is achieved, or rays with a sharp upward launch angle will remain too high when extended to the source range. In our ray-trace procedure, there are rays $1 / 2$ refract initiated at $\left(0 ; z_{r}\right)$ which intersect the Earth's surface (the $\mathrm{x}$-axis) at a range $\mathrm{x}<\mathrm{x}_{\mathrm{t}}$. These rays are terminated in the procedure: there are no reflections at 
Earth-surface. There exists a ray launch angle $\phi_{\min }$ with $-\pi / 2 \leq \phi_{\min }<\pi / 2$ which is the smallest angle resulting in a ray which will intersect the vertical plane.

There are three important elements that are a result of the occurrence of sub-refractive mirages:

1. the existence of a second distinct image of a source point;

2. the orientation (erect vs. inverted) of the image;

3. the local magnification in the image.

All of this information is encoded within a functional dependence of elevation angles at a selected range: $\phi_{\text {geom }}\left(\phi_{\text {refract }}\right)$.The transfer map relationship is a map from the geometric 'atmosphere-free' angular position $\left(\phi_{\text {geom }}\right)$ to the refractive 'apparent' angular position $\left(\phi_{\text {refract }}\right)$. The transfer map is dependent upon the receiver height $z_{\mathrm{r}}$ at range $\mathrm{x}=0$, and it is defined for a vertical plane at the range point $\mathrm{x}=\mathrm{x}_{t}$. It is determined by finding all rays $\rho_{\text {refract }}$ originating at the receiver location $\left(0 ; \mathrm{z}_{\mathrm{r}}\right)$ which intersect a vertical plane at range $\mathrm{x}=\mathrm{x}_{t}$.

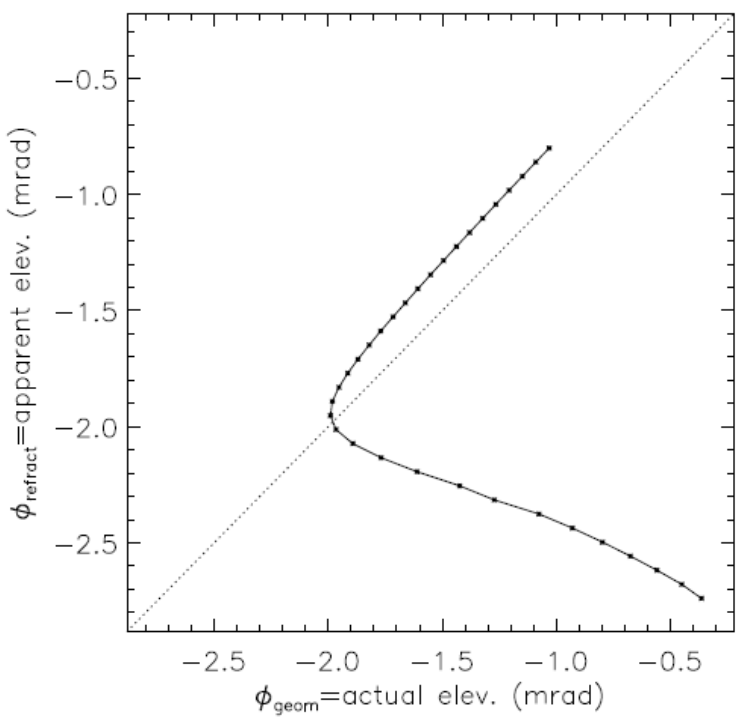

Fig. 4. The transfer map is a transformation from the geometric (or atmosphere-free) actual angular position $\phi_{\text {geom }}$ to the refractive apparent angular position $\phi_{\text {refract }}$. This map is calculated for the ray-trace shown in fig. 1 at a range of 24 $\mathrm{km}$. The dotted-line of $\phi_{\text {refract }}=\phi_{\text {geom }}$ indicates an identity transformation (the atmosphere-free case).

A transfer map for the ray-trace in fig. 1 is shown in fig. 4 . The discrete points represent a continuous curve, and the point of infinite derivative on this curve corresponds to the caustic point in the ray-trace envelope. The three important features noted at the beginning of the section can all be determined from this map. The magnification $\mathrm{d}$ of the image is determined by

$$
\delta=\left|\frac{d \phi_{\text {refrac }}}{d \phi_{\text {geom }}}\right|
$$

and the orientation of the image (erect or inverted) is given by the sign of $d \phi_{\text {refract }} / d \phi_{\text {geom }}$. 
The point at which the graph of the transfer map (fig. 4) has infinite slope corresponds to the caustic point, and this point can also be located on the ray-trace in fig. 1 as the lowest height for all rays intersecting the vertical line at the $24 \mathrm{~km}$ range.

\section{THE REFRACTIVE PROPAGATION FACTOR}

An application of the transfer map calculation is the determination of a "geometric" gain, which is a change in signal intensity due entirely to the nature of the refractive field between target and sensor [3]. The propagation factor $F$ is defined as the ratio between the actual field amplitude at a selected field point and the corresponding field amplitude at that point in free-space propagation conditions. The field intensity at the receiver is given in terms of the field amplitude propagation factor $\mathrm{F}$ by

$$
F^{2}=\sum_{\phi_{\text {refrac }}}\left|\frac{d \phi_{\text {refrac }} / d z}{d \phi_{\text {geom }} / d z}\right|_{(x, z)=\left(x_{t}, z_{t}\right)}
$$

where the summation is over all values $-\pi / 2 \leq \phi_{\text {refract }}<\pi / 2$ for which rays terminate at $\left(x_{t}, y_{t}\right)$. The propagation factor is defined for piecewise horizontally homogeneous environments, and it is dependent only on the spatial locations of the source point and receiver point in space. It is therefore necessary mathematically for the definition to include the full $1 / 4$-radian fan of rays for $\phi_{\text {refract }}$ to ensure that all rays from a source are accounted for at the receiver. Therefore at the upper and lower summation limits, $\phi_{\text {geom }}=\phi_{\text {refract }}=-\pi / 2$ and $\phi_{\text {geom }}=\phi_{\text {refract }}=\pi / 2$ since it is only the vertical rays that are certain to be undeviated in a piecewise horizontally homogeneous propagation environment. In practice, the field of view of a sensor will generally be considerably less than $p$ radians.

A fan of rays that has been calculated for a given refractive profile can be used to deduce the refractive modifications to the observed source intensity. We use a term that is borrowed from radio-frequency propagation models, and call this multiplicative term the refractive propagation factor. The word "refractive" is appended to indicate that changes in field amplitude due to refractive effects are included, but not changes due to any reflection of the propagating beam. The refractive propagation factor is a function of both the receiver location and the transmitter location. The receiver is located by definition at zero range, so the propagation factor is a function of three parameters: receiver height $z_{\mathrm{r}}$, transmitter height $z_{\mathrm{t}}$, and range $x_{\mathrm{r}}$.

Given the receiver height $z_{\mathrm{r}}$, a two-dimensional field of values for $F^{2}$ is determined. At a range of $\approx 5 \mathrm{~km}$ the refractive propagation factor is $F^{2} \approx 1$, because the field magnification is nearly neutral (see fig. 1). After a longer propagation path, a sub-refractive mirage develops, and a second image of the transmitter becomes visible. The combined intensities from the two images result in a signal intensity greater than the freespace value. This is indicated by fig. 5 which shows a calculation of the gain-height function at a range of $24 \mathrm{~km}$. The ray-trace foundation for this calculation can be seen in fig. 1 where a vertical dotted line indicates the vertical plane at a range of $24 \mathrm{~km}$. In these conditions a sub-refractive mirage is visible. The plot indicates that a transmitter at a height of $11 \mathrm{~m}$ will not be visible, while a transmitter at $13 \mathrm{~m}$ will be detected with a strongly amplified signal. 


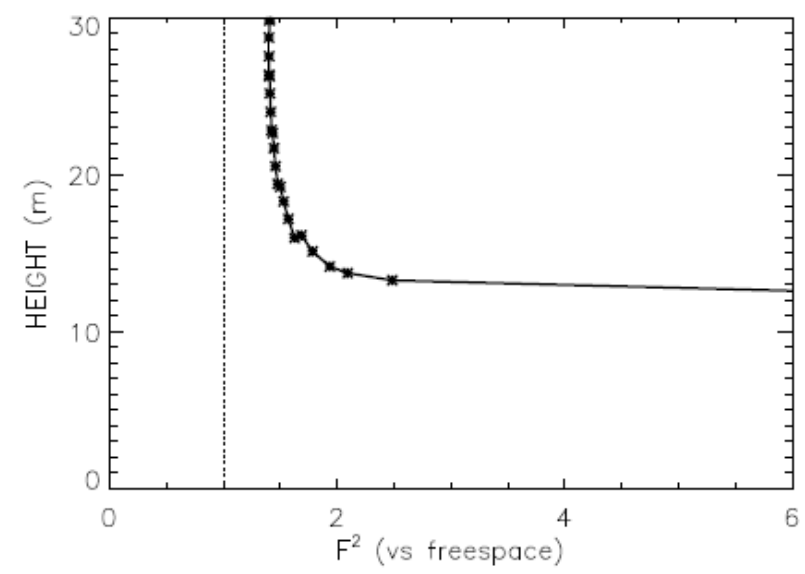

Fig. 5. Gain as a function of height at a range of $\sim 24 \mathrm{~km}$. Note the large increase in gain at $\approx 13 \mathrm{~m}$ height. Below $12 \mathrm{~m}$ there is zero signal.

The height-gain curve implies that $F^{2}>1$ for $z>13 \mathrm{~m}$, and as height decreases from $30 \mathrm{~m}, F^{2}$ increases. As height is reduced to $\approx 13 \mathrm{~m}, F^{2}$ continues to increase and becomes divergent at $z_{c} \approx 12.5$. This is because of the source point approach to a singularity at the caustic at a range of $24 \mathrm{~m}$. This can be verified by reference to fig. 1 for which the caustic point is at the intersection of the lowest point of the ray envelope and the vertical line at range $24 \mathrm{~km}$. There are methods for resolving the singularities at the caustic $[4,5]$ but these methods are not used here.

\section{CONCLUSIONS}

We have described the geometric optics approach to the problem of characterizing sources or targets at long range within the marine atmospheric surface layer. In this environment, sub-refractive conditions are common and depending upon the sensor-target geometry, inferior mirages of the target can occur at the sensor. The elevation angle of the two images can be used to deduce height and range data for the target. An atmospheric surface layer for which the air-sea temperature difference is negative exhibits a crucial feature: the rays form a local coordinate system starting at some point downrange. The logarithmic temperature profile ensures that lower elevation rays are detected to intersect upper elevation rays.

The existence of a locally non-degenerate coordinate system implies that in some region of range-height space there exists a one-to-one correspondence with an upper elevation-lower elevation pair that is unique to that point.

A second consequence of the calculation within the geometric optics approach is the capability to compute a geometric gain induced by the refractivity field. This refractive propagation factor is a necessary first-order component in a complete model for near-surface horizontal path infrared or optical transmission. The refractive effects are shown in the example to be substantial determinants of the received signal intensity. The refractive propagation factor is a multiplicative quantity derived entirely from the local refractivity field and the geometry of the complete propagation system.

The ray method also provides a useful framework for the calculation of other path-dependent signal metrics such as molecular and aerosol extinction, scintillation and the refractive index structure function.

\section{REFERENCES}

1. Frederickson, P., K. Davidson, and A. K. Goroch "Operational Bulk Evaporation Duct Model for MORIAH", NPS/MR Technical Report, NPS/MR-2000-002, 2000

2. Lehn, W., Appl. Math. Model. 9, 447, 1985. 
3. Doss-Hammel, S. M., Zeisse, C. R., Barrios, A. E., de Leeuw, G., Moerman, M., de Jong, A. N., Frederickson, P.A., Davidson, K. L., "Low-Altitude Infrared Propagation in a Coastal Zone: Refraction and Scattering", Applied Optics, 41, $3706\{3724,2002$.

4. Kravtsov, Y., and Orlov, Y., Geometrical Optics of Inhomogeneous Media, Springer-Verlag Berlin, 1990.

5. Kravtsov, Y., and Orlov, Y., Caustics, Catastrophes and Wave Fields, Springer-Verlag Berlin, 1999.

6. Trahan, J., and Rivera, H. "Mid-wave infrared low elevation propagation experiment on the Potomac River, Summer 1995", Technical Report NSWCDD/TR-96/23 (Sept. 1996).

7. N. Platt, S. Hammel, J. Trahan, H. Rivera, "Mirages in the marine boundary layer - comparison of experiment with model", Proc. IRIS Passive Sensors, Monterey, CA, vol. 2, 195-210, 1996. 